\title{
A case of hypokalaemic periodic paralysis
}

\author{
S Jeyabalan ${ }^{1}$, Lilanthi de Silva ${ }^{2}$, S Sutharshini ${ }^{3}$, S Jasintha ${ }^{3}$
}

Sri Lanka Journal of Child Health, 2012; 41(2): 95-96

(Key words: Hypokalaemia; paralysis; weakness; potassium)

\section{Introduction}

Hypokalaemic periodic paralysis is the most common form of periodic paralysis, but is still quite rare, with an estimated prevalence of 1 in $100,000^{1}$. It may be familial with autosomal dominant inheritance or may be acquired in patients with thyrotoxicosis ${ }^{2}$. In the familial form, clinical penetrance is often incomplete, especially in females ${ }^{3}$. The disorder is three to four times more commonly clinically expressed in males. Approximately one-third of cases represent new mutations ${ }^{4}$. It occurs due to mutation in one of various genes responsible for voltage gated channels in muscle cells ${ }^{5}$. Mutation in $\mathrm{Ca}$ and $\mathrm{Na}$ channel genes are common in this rare form of channelopathy ${ }^{6}$.

\section{Case report}

This is an 11 year old girl born to non consanguineous healthy parents. She was apparently well until 6 years of age with normal development and growth. Initial presentation was at the age of six years with acute onset of weakness involving both upper and lower limbs. The limbs were symmetrically flaccid. Reflexes were diminished but the sensory functions were intact. Higher functions were not affected. Bladder and bowel sensation were intact. Thereafter child presented with similar recurrent episodes. There was no respiratory muscle involvement or swallowing difficulties. There was no history of any preceding febrile illness. However, most of the episodes occurred in the early morning following a prolonged rest at night.

In the detailed history, most of the episodes were precipitated by certain foods such as eating cake or ice-cream and exertion followed by rest. Initially recurrence was infrequent but after attaining puberty there was a marked increase in recurrence rate. There was no family history of similar illnesses. All episodes were associated with hypokalaemia. Her serum potassium levels were in

\footnotetext{
${ }^{1}$ Senior Registrar in Paediatrics, ${ }^{2}$ Consultant paediatrician, ${ }^{3}$ Registrar in Paediatrics, North Colombo Teaching Hospital, Ragama
}

(Received on 8 June 2011: Accepted after revision on 26 August 2011) the range of 2-3 $\mathrm{mmol} / \mathrm{l}$ at presentation of each episode. Other biochemical investigations during the episodes were unremarkable.

Each episode was treated with oral potassium tablets and her symptoms and signs disappeared within a few hours. The child was extensively investigated during the first episode including nerve conduction studies, electromyogram and thyroid function tests. All results were within normal limits. Later, the child was prescribed spironolactone combined with dietary advice and there was a marked reduction in the recurrence rate.

\section{Discussion}

In hypokalaemic periodic paralysis symptoms often begin in early childhood but can occur in infancy or adolescence. However, the disease is nearly always symptomatic by ten years of age ${ }^{5}$ In this child the presentation was at 6 years of age. Weakness or paralysis occurs transiently and is mostly symmetrical but can be asymmetrical ${ }^{7}$. In our patient there was symmetrical involvement of both upper and lower limbs. Proximal muscles are mainly involved but rarely trunk muscle, respiratory muscle and cardiac muscle can be involved. In this child there was no respiratory or cardiac muscle involvement. Sensation is not affected $^{7}$. In our patient the sensory level was intact. Initially episodes are infrequent and gradually become more frequent and ultimately daily $^{8}$. In this child initially recurrence was infrequent but after attaining puberty there was a marked increase in recurrence rate. Whilst this condition is usually inherited as an autosomal dominant trait ${ }^{2}$, there was no family history in this instance.

In hypokalaemic periodic paralysis attacks are triggered by strenuous exercise followed by rest, high carbohydrate meals, meals with high sodium content, sudden changes in temperature, and even excitement, noise or flashing lights ${ }^{9}$. In our patient precipitating factors included foods such as cake or ice-cream and exertion followed by rest.

Diagnosis of this condition is mainly clinical supported by laboratory findings of low serum potassium levels. In our patient the serum potassium levels were in the range of $2-3 \mathrm{mmol} / 1$ at presentation of each episode. The Compound 
Muscle Amplitude Potential (CMAP) test, also called the exercise EMG, is diagnostic in $70-80 \%$ of cases when done correctly 9 . This was not done in our patient. Muscle biopsy in between attacks is normal but during the symptomatic period vacuolar myopathy can be demonstrated ${ }^{5}$. Muscle biopsy was not performed in our patient. Molecular genetic testing is available but is fairly expensive and was not resorted to in this child.

Management of this condition, during acute episodes needs potassium supplements. In this child each episode was treated with oral potassium tablets and her symptoms and signs disappeared within a few hours.

To prevent recurrences the following are useful:

1. Avoid carbohydrate rich foods and promote slow release carbohydrate diet.

2. Avoid strenuous exercise.

3. Low salt diet.

4. Daily use of carbonic anhydrase inhibitors such as acetazolamide and aldosterone antagonists such as spironolactone will help to reduce frequency of recurrences ${ }^{3}$.

Our patient was prescribed spironolactone combined with dietary advice and there was marked reduction in the recurrence rate.

Prognosis of this condition is as follows: initially in-between attacks child is normal with normal muscle function ${ }^{3}$. However, in $50 \%$ of patients, progressive myopathy with permanent weakness, even in between attacks, can occur ${ }^{7}$. Their life span is expected to be normal but rarely death can occur during acute episode due to cardiac arrhythmias or respiratory muscle involvement ${ }^{7}$.

\section{References}

1. Fontaine B. Periodic paralysis. Adv Genet 2008; 63:3.

http://dx.doi.org/10.1016/S0065$\underline{\text { 2660(08)01001-8 }}$
2. Lin SH. Thyrotoxic periodic paralysis. Mayo Clin Proc 2005; 80(1):99. http://dx.doi.org/10.4065/80.1.99

3. Elbaz A, Vale-Santos J, Jurkat-Rott K, Lapie P, Ophoff RA, Bady B, et al. Hypokalaemic periodic paralysis and the dihydropyridine receptor (CACNL1A3): genotype/phenotype correlations for two predominant mutations and evidence for the absence of a founder effect in 16 caucasian families. Am J Hum Genet 1995; 56(2):374.।

4. Venance SL, Cannon SC, Fialho D, Fontaine B, Hanna MG, Ptacek LJ, et al. The primary periodic paralyses: diagnosis, pathogenesis and treatment. Brain 2006; 129(Pt 1):8.

5. Behrman RE, Kliegman RM, Jensen HB, editors. Nelson textbook of pediatrics. $17^{\text {th }}$ edition. Philadelphia: Saunders, 2004.

6. Barohn RJ. Muscle diseases. In: Goldman L, Ausiello D, eds. Cecil Medicine. 23rd ed. Philadelphia, Pa: Saunders Elsevier; 2007:chap 447.

7. McIntosh N, Helms PJ, Smyth RL, editors. Forfar \& Arneil's Textbook of pediatrics, $6^{\text {th }}$ edition, London; Churchill Livingstone, 2003.

8. Gerald M. Fenichel, editor. Clinical paediatric Neurology, 5th edition. Saunders, 2005.

9. Hypokalaemic periodic paralysis. Available from:

http://en.wikipedia.org/wiki/Hypokalemic_peri odic_paralysis 